\title{
Evaluating the Polarization of
} Tumor-Associated Macrophages Into M1 and M2 Phenotypes in Human Cancer Tissue: Technicalities and Challenges in Routine Clinical Practice

\begin{abstract}
Sharmilla Devi Jayasingam ${ }^{1}$, Marimuthu Citartan ${ }^{2}$, Thean Hock Thang ${ }^{2,3}$, Anani Aila Mat Zin ${ }^{4}$, Kai Cheen Ang $^{2}$ and Ewe Seng Ch'ng ${ }^{1 *}$
\end{abstract}

\section{OPEN ACCESS}

Edited by:

Mei Lan Tan,

University of Science, Malaysia

Reviewed by:

Till Adhikary,

University of Marburg, Germany

Guoguang Zheng,

Chinese Academy of Medical

Sciences and Peking Union Medical

College, China

Rathindranath Baral,

Chittaranjan National Cancer

Institute, India

${ }^{*}$ Correspondence:

Ewe Seng Ch'ng

eschng@usm.my;

chngeweseng@hotmail.com

Specialty section:

This article was submitted to

Radiation Oncology,

a section of the journal

Frontiers in Oncology

Received: 29 August 2019 Accepted: 16 December 2019 Published: 24 January 2020

Citation:

Jayasingam $S D$, Citartan $M$

Thang TH, Mat Zin AA, Ang KC and Ch'ng ES (2020) Evaluating the

Polarization of Tumor-Associated

Macrophages Into M1 and M2 Phenotypes in Human Cancer Tissue:

Technicalities and Challenges in

Routine Clinical Practice.

Front. Oncol. 9:1512

doi: 10.3389/fonc.2019.01512
${ }^{1}$ Oncological and Radiological Sciences Cluster, Advanced Medical and Dental Institute (AMDI), Universiti Sains Malaysia, Kepala Batas, Malaysia, ${ }^{2}$ Infectious Disease Cluster, Advanced Medical and Dental Institute (AMDI), Universiti Sains Malaysia, Kepala Batas, Malaysia, ${ }^{3}$ Faculty of Applied Sciences, AIMST University, Kedah, Malaysia, ${ }^{4}$ Department of Pathology, School of Medical Sciences, Universiti Sains Malaysia, Kubang Kerian, Malaysia

Tumor-associated macrophages (TAMs) as immune cells within the tumor microenvironment have gained much interests as basic science regarding their roles in tumor progression unfolds. Better understanding of their polarization into pro-tumoral phenotype to promote tumor growth, tumor angiogenesis, immune evasion, and tumor metastasis has prompted various studies to investigate their clinical significance as a biomarker of predictive and prognostic value across different cancer types. Yet, the methodologies to investigate the polarization phenomena in solid tumor tissue vary. Nonetheless, quantifying the ratio of M1 to M2 TAMs has emerged to be a prevailing parameter to evaluate this polarization phenomena for clinical application. This mini-review focuses on recent studies exploring clinical significance of M1/M2 TAM ratio in human cancer tissue and critically evaluates the technicalities and challenges in quantifying this parameter for routine clinical practice. Immunohistochemistry appears to be the preferred methodology for M1/M2 TAM evaluation as it is readily available in clinical laboratories, albeit with certain limitations. Recommendations are made to standardize the quantification of TAMs for better transition into clinical practice and for better comparison among studies in various populations of patients and cancer types.

Keywords: tumor-associated macrophages, M1, M2, immunohistochemistry, CD68, CD163, cancer

\section{INTRODUCTION}

Macrophages are a group of differentiated immune cells that are phagocytic in nature and have specific phenotypic characteristics (1). They are a diverse set of immune cells which are polarized by various microenvironmental stimuli to generate a heterogeneous population with different properties and functions. Macrophages are involved in tissue homeostasis, defense mechanisms, and wound healing. They also play a role in various diseases, such as autoimmune disorders, atherosclerosis, and tumorigenesis $(1,2)$. 
Activated macrophages are often classified as proinflammatory M1 macrophages or anti-inflammatory M2 macrophages. The M1-like phenotype is induced by toll-like receptor ligands (bacterial lipopolysaccharide) or Th1 cytokines, such as tumor necrosis factor alpha (TNF- $\alpha$ ), interferon gamma (IFN $\gamma)$ and colony stimulating factor 2 (CSF2) (3, 4). M1-like macrophages exert high antigen presenting capacity (5). They also secrete reactive oxygen species (ROS) and cytokines like IL-6, IL-12, IL-23, and TNF- $\alpha$, associated with microbicidal and pro-inflammatory activities (6). Thus, they are termed as the "fight" macrophages and are associated with good prognosis in cancer context $(7,8)$.

M2-like macrophages, on the other hand, are polarized by Th2-derived cytokines such as IL4, IL10, IL13, transforming growth factor beta (TGF $\beta$ ) or prostaglandin E2 (PGE2) (3). They are known as the "repair" or "fix" macrophages as they promote tissue repair via immune tolerance and tissue remodeling, debris scavenging and immune modulation (7). When it comes to cancer, M2-like macrophages support angiogenesis by secreting adrenomedullin and vascular epithelial growth factors (VEGFs) and express immunosuppressive molecules such as IL10, programmed death-ligand 1 (PD-L1), and TGF $\beta$, favoring tumor growth (3). They are regarded as "friends" by cancer cells.

It should be noted that the actual polarization state of macrophages is far more complex than the simple binary M1, M2 classification, which serves to define only macrophage functions. In fact, macrophages are highly plastic cells consisting of a spectrum of activation states, with M1 and M2 representing the extremes on each opposing end. Many of the subsets display mixed heterogeneity and some are yet to be discovered or fully characterized. Detailed explanation on macrophage polarization and the mechanisms has been described in a number of recent reviews $(1,3,9)$ and thus will not be further elaborated here.

A specific class of macrophages, tumor-associated macrophages (TAMs) are macrophages within the tumor microenvironment (TME). TAMs regulate metastasis by producing growth factors, cytokines, and other molecules. In recent years, researchers have been investigating TAMs as a therapeutic strategy to curb tumor progression and metastasis (9).

\section{CLINICAL SIGNIFICANCE OF TUMOR-ASSOCIATED MACROPHAGES}

As a whole, TAMs play a crucial role in cancer progression as supported by many in-vitro and in-vivo studies (10-12). In parallel to the growing insights into the roles of TAMs, many studies have been conducted to look into the clinical significance of TAMs in solid tumors. Generally, higher densities of TAMs are often observed in more advanced tumor stages as evidenced in esophageal cancer (13), ovarian cancer (14), breast cancer (12), and pancreatic cancer (15). Additionally, the negative impact of TAMs on patients' overall survival was also reflected in esophageal cancer (13), pancreatic cancer (16), breast cancer (17), lung cancer (18), and gastric cancer (19).

\section{M2 TUMOR-ASSOCIATED MACROPHAGES-THE "AGGRESSOR" OF TUMOR}

Higher infiltration of the M2 TAMs is associated with a more aggressive tumor characteristic, reflected by tumor invasion, progression, and metastases. A recent study on non-functional pituitary adenomas (NFPAs) discovered that the cultured M2 macrophages significantly enhanced the proliferation and invasion of the primary NFPA cultures as compared to the cultured M1 macrophages (20). In hepatocellular carcinoma, higher M2 TAMs were strongly correlated with more tumor number and advanced stages (21). Similarly, M2 infiltration in breast cancer was markedly correlated with larger tumor size, advanced stages, and angiogenesis. Polarization toward the M2 phenotype showed strong correlation with the aggressiveness of breast cancer characterized by higher histologic grade, higher Ki-67 proliferating index, estrogen receptor and progesterone receptor negativity $(22,23)$.

TAMs are also related to resistance toward cancer treatments. M2 macrophages can induce chemoresistance by secreting growth factors and inhibiting cell death signaling pathways in tumor cells, thus sheltering them from the chemotherapy effects (24). Clinical studies have demonstrated that a high number of M2 TAMs in tumor cause chemoresistance and radioprotective effects, leading to therapy failure and poor prognosis in patients (25-27). One study discovered that TAMs skewed toward the M2 phenotype resulted in immuno-tolerance and resistance to anti-Her2/neu therapy in breast cancer. When the antiHer2/neu therapy was combined with targeted delivery of IL21, a cytokine that can enhance phagocytosis and protease activity of macrophages, TAM polarization was skewed toward the M1 phenotype instead, thus reversing immunosuppression and resuming tumoricidal activity (28). Hence, higher M2 TAMs were associated with treatment resistance, whereas higher M1 TAMs correlated with improved treatment outcome.

\section{M1/M2 RATIO AS THE MORE AMENABLE MEANS OF CANCER PROGNOSTICATION IN CLINICAL PRACTICE}

The accentuation on the usage of M1/M2 ratio in prognosticating cancer is supported by the ambiguities concerning the sole densities of TAMs in patients' prognosis $(29,30)$. For instance, although TAMs in general have negative effect on the gastric patients' prognosis in many studies $(19,31,32)$, some studies showed opposing results (33-35). This contradiction is mainly because most studies only involve the total number of macrophages (M1 + M2), instead of evaluating M1 and M2 TAMs separately. In order to resolve these disputes, researches have shown that polarization of TAMs in cancer, more specifically the $\mathrm{M} 1 / \mathrm{M} 2$ ratio, is a more biologically relevant indicator to prognose cancer as compared to whole TAM densities $(36,37)$. This ratio could represent either a positive or a negative impact on tumor growth $(14,29,38)$. 
A higher $\mathrm{M} 1 / \mathrm{M} 2$ ratio in cancer tissue usually signifies a favorable outcome. Petrillo et al. (39) demonstrated that in locally advanced cervical cancers (LACC), a more frequent complete pathological response to chemotherapy (CT) or radiotherapy (RT) was observed in patients with high $\mathrm{M} 1 / \mathrm{M} 2$ ratio. On the other hand, polarization of TAMs toward the M2 phenotype as reflected by lower M1/M2 ratio was an independent predictor for poor response to CT or RT and shorter survival in LACC (39). This was supported by other studies involving different cancer types such as ovarian cancer (40), pediatric classical Hodgkin lymphoma (cHL) (41), and multiple myeloma (42).

A lower M1/M2 ratio often indicates poor prognosis in cancer patients, while better prognosis is associated with higher M1/M2 ratio. For instance, in LACC, the tumor microenvironment is mainly skewed toward the M2 phenotype upon diagnosis. Among these patients, those with high $\mathrm{M} 1 / \mathrm{M} 2$ ratio exhibited a longer 5-years-disease-free (67.2 vs. $44.3 \% ; P=0.019)$ and 5 years overall survival (OS) compared to cases with low M1/M2 ratio (69.3 vs. $46.9 \%, P=0.037$ ) (39). Similarly, in gastric cancer, 27 out of 52 radically resected gastric patients with $\mathrm{M} 1$ density above the median had a significantly higher survival rate as compared to those below the median (median survival of 25.6 months vs. 17.6 months, $P=0.041$ ). Moreover, 26 patients with $\mathrm{M} 1 / \mathrm{M} 2$ ratio above the median showed median OS of 27.2 months as compared to 15.5 months of the patients below the median (37). Hence, the M1 macrophage density and M1/M2 ratio are important factors in predicting patients' survival time.

\section{EVALUATION OF M1 AND M2 POLARIZATION IN TISSUE SECTION: TECHNICALITIES}

It is evident that the effort to harness TAMs as a biomarker in clinical practice has shifted from simple enumeration of TAM densities to specifically characterizing the vector of polarization phenomena by employing M1/M2 ratio as a surrogate marker (37, 39, 43). In this transition, several technicalities and challenges are worth further scrutinization.

The field of immunology has several advancements in accessing TAMs in tissue biopsies. Among them are flow cytometry, gene expression profiling, and the recent highthroughput single-cell sequencing (44-46). These advancements are no doubt welcomed in the research field as they are more standardized and quantitative in nature. However, when it comes to routine clinical diagnosis where cost, feasibility, and time are restricting factors, the immunohistochemistry is the preferred method. What makes immunohistochemistry distinctive is the potential to assess a cell expression in its own microenvironment (47). Visual details on protein distribution and localization can be procured without the need to destroy the histologic architecture of a tissue (48). Accordingly, studies focusing on TAM characterization in solid clinical samples often adopt immunohistochemistry in their methodologies till date $(8,49,50)$. In contrast, methods such as flow cytometry and gene expression are performed in vitro and could not reflect the complexity of immune responses observed in vivo
(51, 52). Hence, when it comes to routine clinical practice, immunohistochemistry of histological section remains the best platform to study TAMs in situ and is therefore, the sole emphasis of this review. To expound immunohistochemistry in the context of technicality, there are several important issues to consider.

\section{Tumor-Associated Macrophages Markers}

Immunohistochemical markers used to identify M1 and M2 TAMs are the cornerstones of TAM evaluation. Immunohistochemistry protocol varies greatly in selecting the best markers to quantify TAMs. Unlike M2, M1 macrophages highly express HLA-DR (53) and inducible nitric oxide synthase (iNOS) in inflamed human tissue $(54,55)$. Meanwhile, pSTAT1, the phosphorylated form of STAT1, is a transcription factor which promotes M1-associated functions (56). Thus, some common markers for M1 TAMs in human samples are HLA-DR, iNOS, and pSTAT1. In contrast, common markers for M2 TAMs are CD206, CD204, and CD163, attributable to the high expression of the mannose receptor-1 (CD206) and macrophage scavenger receptors (CD204 and CD163) by the M2 TAMs (Figure 1) $(57,58)$.

Immunohistochemistry also differs in the number of markers employed to identify each population of TAMs. In some studies, only a single marker is used to identify M1 or M2 TAMs, such as CD68 and CD163 (59-61). The single marker-based practice is not encouraged as this affects reproducibility, makes case to case comparison difficult, and the antigens can also be co-expressed by other cells (62). Moreover, dependency on a single marker to evaluate TAMs followed by correlation with clinicopathological findings may yield contradictory results, as reflected by some papers $(29,32,35)$. Therefore, the trend has now shifted to double immunohistochemistry staining to better characterize M1 and M2 TAMs. Some of the frequent double stain combinations are CD68/iNOS $(63,64)$ and CD68/HLA-DR $(65,66)$ for M1 TAMs and CD68/CD163 for M2 TAMs (23, 65, 66). However, problems may arise if the antigens chosen are co-localized in the same cell compartment. For example, combining CD68/iNOS for M1 TAMs may be difficult to interpret as both markers are localized in the cytoplasm. This poses the risk of causing one color to overshadow the other or resulting in a mixture of colors, complicating visual interpretation (43). The best practice is to select the combination whereby the individual antigens are confined to unique, non-overlapping cellular locations to permit precise visual interpretation.

\section{Detection Methods}

Another important element in immunohistochemistry is choosing the appropriate detection method. Immunohistochemistry is categorized into two main detection methods; immunofluorescence and the chromogenic method. The principles for both methods are similar; the specific antigenantibody reaction is probed by a labeled antibody to localize the target antigens. This label could either be a fluorochrome or an enzyme/chromogen (67). By convention, the chromogenic method is usually referred as immunohistochemistry (IHC) without specification, while the fluorochrome detection method is known as immunofluorescence (IF). 


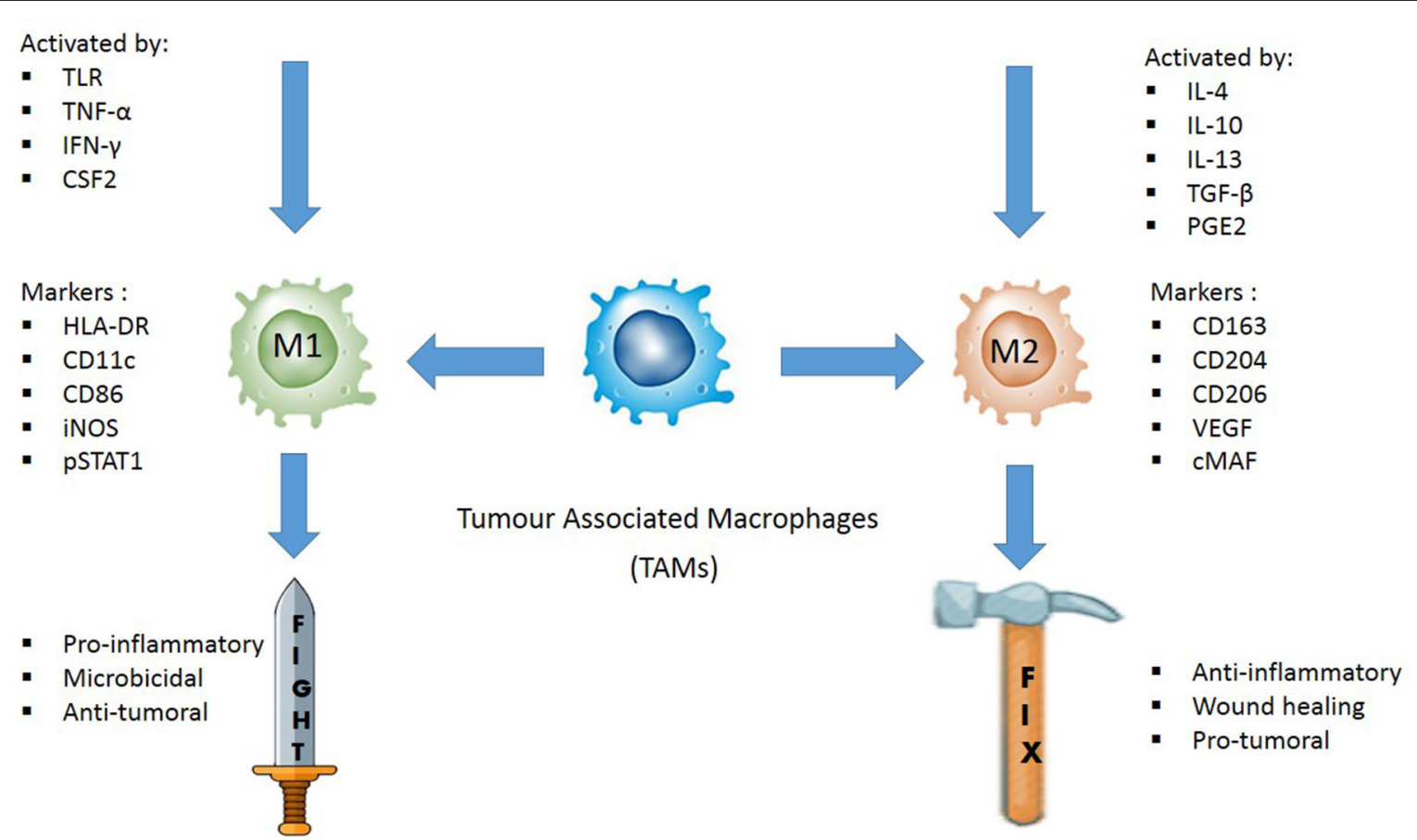

FIGURE 1 | Illustration of how M1 and M2 TAMs are activated, their respective functions, and the common IHC markers used to distinguish the two phenotypes in tissue samples. M1 TAMs are activated by toll-like receptors (TLRs) or Th1 cytokines, such as tumor necrosis factor alpha (TNF- $\alpha$ ), interferon gamma (IFN $\gamma$ ), and colony stimulating factor 2 (CSF2). They are pro-inflammatory, microbicidal and have anti-tumoral effect. HLA-DR, CD11c, CD86, iNOS, and pSTAT1 are some of the common IHC markers used to identify M1 TAMs. M2 TAMs are activated by IL4, IL10, IL13, transforming growth factor beta (TGFB) or prostaglandin E2 (PGE2). They are anti-inflammatory, promotes wound healing, and are pro-tumoral. CD163, CD204, CD206, VEGF, and CMAF are some of the IHC markers used to distinguish M2 TAMs.

A scoping search was performed in major databases to assess the application of IHC and IF in quantifying M1 and M2 TAMs in tumorous tissue by retrieving articles from the last 6 years (20142019) with the inclusion criterion of human tumor samples. Cell lines and animal models were excluded. Based on the analysis, more than $80 \%$ of the papers chose IHC over IF, while IF was mostly performed on cell lines and animal models (Table $\mathbf{1}$ ).

In routine clinical settings, IHC is mainly preferred over IF due to the convenience of the formalin-fixed paraffin embedded (FFPE) tissue, i.e., huge amounts of archived clinical specimens owing to the simplicity and low cost (77). Furthermore, a fluorescence microscope is not required; a simple light microscope would suffice to view the IHC stain. The stained samples can also be stored for a long time as the chromogenic stains are more resistant to photobleaching. Hence, IHC using FFPE tissue blocks has high importance in retrospective studies.

Hitherto, IF is not routinely used in clinical settings due to several limitations. Firstly, IF usually requires a fresh frozen tissue where the proteins are preserved in their native state. Fixation with formalin for instance could be harsh on the tissue, blocking the epitopes of the target antigen or altering them. Blockade of the epitopes impairs their recognition by antibodies, resulting in poor signal production. There is also the risk of autofluorescence in IF if FFPE tissue was used
(48). Besides, frozen tissue could prove to be costly, as it requires specialized equipment to maintain the samples (78). Furthermore, IF necessitates a designated, cool, and darkened room for the slides to be read as IF is highly susceptible to photo bleaching. This could be inconvenient in cases where re-reading of the slides is necessary.

\section{Localization of Tumor-Associated Macrophages: Tumor Stroma vs. Tumor Nests}

One main reason for the discrepancy in TAMs effect on clinicopathological parameters is the location of TAM infiltration. TAMs in different tumor compartments may have a different implication on cancer prognosis. For example, in breast cancer studies, infiltration of M2 TAMs in tumor stroma instead of tumor nests was associated with larger tumor size, higher histological grade, higher 5-year recurrence, and 5-year breast cancer mortality, in addition to being an independent predictor of recurrence-free survival (RFS) and OS $(23,79)$. Similarly, in esophageal carcinoma, infiltration of M2 TAMs in tumor stroma was strongly associated with more malignant characteristics such as metastasis and clinical stage progression. This highlights the importance of considering the localization of TAMs in addition to their number as a prognostic marker. 
TABLE 1 | List of articles which evaluated M1 and M2 TAMs in tissue sections from 2014 to 2019.

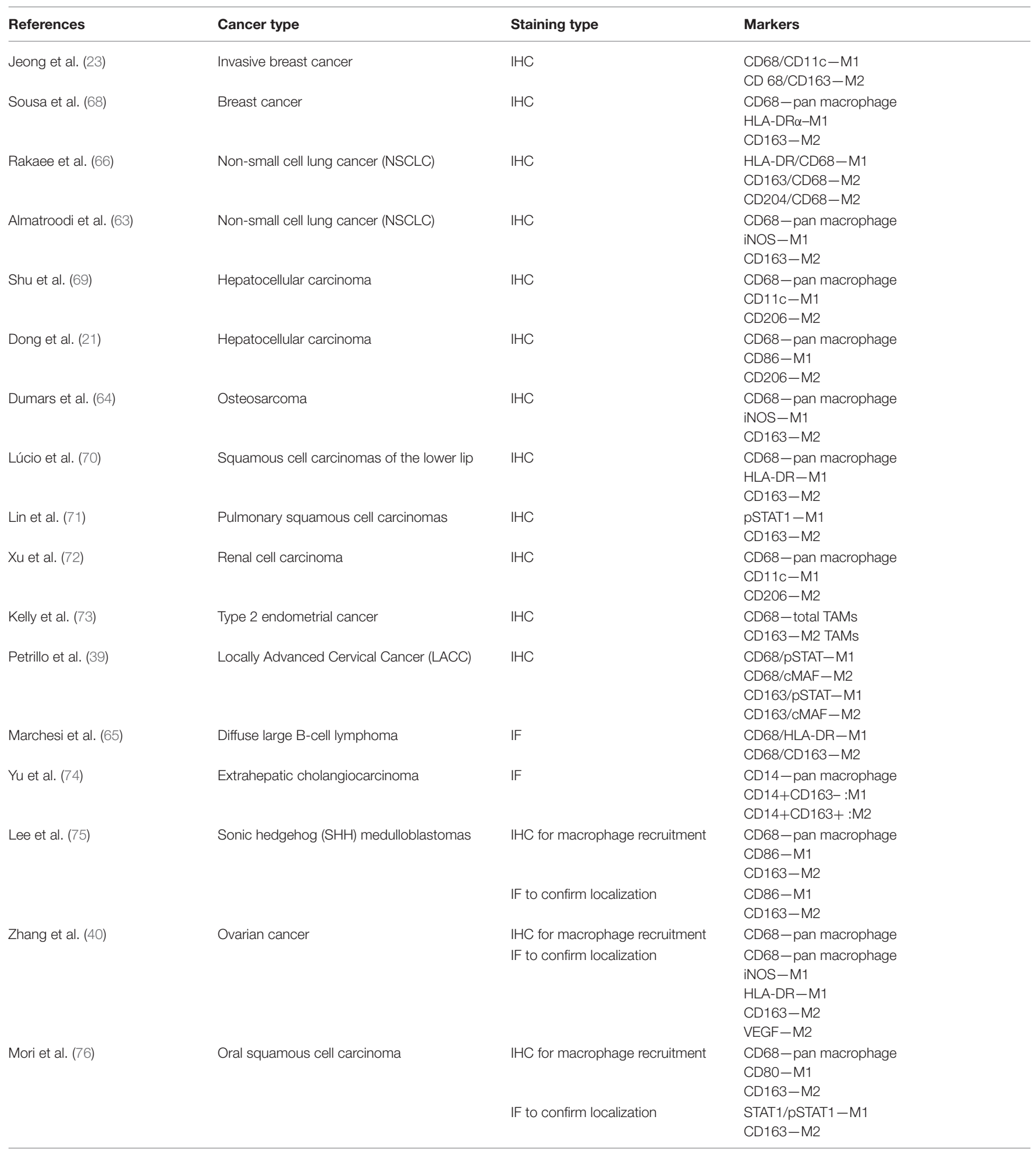

\section{Quantification of Tumor-Associated Macrophages}

Most researches often employ the "hotspot" quantitative method to evaluate TAMs. However, this method varies in the number of fields selected for TAM evaluation and the counting method performed. In some studies, 10 "hot" fields were chosen to quantify TAMs before obtaining an average, while others settled with three or five "hot" fields. As for counting TAMs, certain 
papers applied software such as ImageScope and ImageJ Cell, while others consulted two pathologists with vast experience to quantify TAMs manually $(23,39,69,70,75)$.

\section{CHALLENGES AND STRATEGIES TO ADDRESS THE LIMITATIONS IN THE EVALUATION OF M1 AND M2 POLARIZATION}

\section{Poor Standardization of Tumor-Associated Macrophage Quantification}

Regardless of IHC or IF, a persisting disadvantage of the immunohistochemistry is the obvious lack of standardization in TAM assessment. Discrepancies in TAM assessment stem from the variation in tissue collection, tissue fixation, tissue thickness, assessment criteria, staining process, and image analysis. One prominent example is the variation in selecting the best markers to quantify TAMs. Different markers are used across different studies as reflected in Table 1. Inconsistencies in TAM assessment are more evident between laboratories. Thus, reproducibility is significantly low when the same specimen is evaluated by different laboratories (44). Although concerns have been raised regarding low reproducibility of the immunohistochemistry for the past decades, till date, only minimal efforts have been taken to improve its quality $(44,47,80,81)$. Absolute standardization in immunohistochemistry protocol is difficult; however, small steps taken gradually will eventually lead to a more robust and global operating procedures. For starters, all laboratories should adhere to a fixed section thickness for TAM assessment. In quantifying M1 and M2 TAMs, all researchers/pathologists should reach a consensus of using 10 "hot" spots for a better reflection of the TAM number.

\section{Lack of Robust Marker Confounds the Quantification of Tumor-Associated Macrophages}

Another concerning matter is the need for a highly specific yet flexible marker. For instance, antibodies for CD68, the pan macrophage marker, are not specific for cells of the monocyte/macrophage system besides being immunohistochemically detectable in a variety of other cell types (82). iNOS, a popular M1 macrophage marker, is co-expressed by endothelial cells and arterial wall smooth muscle cells (37). Moreover, there are controversies regarding its use due to the difference in expressions in murine and human macrophages $(83,84)$. CD163, a M2 macrophage marker, is also expressed in some dendritic and endothelial cells (60). This could result in inaccurate M1 and M2 TAM counts. One way to minimize false positive count is to amalgamate quantification of TAMs with the visualization of cell morphology.

The complex nature of macrophage heterogeneity undermines many clinical studies. Although the application of M1/M2 TAM ratio in tumorous tissue has provided meaningful insights into cancer prognosis, it does not reflect the exact functions of these cells; macrophages in vivo exist in a continuum of functional states and may display both M1 and M2 markers, making it difficult to interpret their impact (85). To address this complication, methods like gene expression, cytokine profiling, or miRNA expression can be used to verify their polarization and functions. Nevertheless, the best solution would be the discovery of novel markers which truly reflect TAM heterogeneity. There will be higher possibility for this discovery when the conundrum of macrophage classification is resolved.

\section{Variation in Interpretation}

In immunohistochemistry, it is difficult to address the variability in human perception for its visual analysis (48). Hence, quantification of M1 and M2 TAMs in a tissue section is prone to bias and inter-observation $(5,86)$. Digital image analysis has been introduced to reduce subjective/human error. With this technology, the M1 and M2 TAM count could be automated, ensuring precise and accurate result each time. However, this application could wrongly misinterpret cells such as dendritic and endothelial cells as TAMs. Therefore, a software which can integrate both the automated count and cell visualization to ascertain cell type can yield more reliable and specific TAM count. Although not meant for TAMs, softwares have been developed to enhance the microscopic image analysis. The ImmunoRatio software for example, segments immunostained and hematoxylin-stained cellular areas from the user-submitted image and calculates the labeling index for ER, PR, and Ki-67 in breast cancer (87). With some modifications, it is conceivable that this type of software can be applied for quantification of M1/M2 ratio in solid tumor in future.

\section{CONCLUSION}

The ultimate goal of every pathologist or researcher is to be able to evaluate M1 and M2 TAMs in the most specific and standardized manner for a clear-cut determination of the TAM polarization. For IHC to be considered as a "top-rate" biomarker, optimization of all aspects of IHC is critically needed to develop a universal protocol which is reliable and reproducible for the determination of the polarization of TAMs. This mini review provides caveats as well as insights to better handle this important progressive aspect of solid tumor biology.

\section{AUTHOR CONTRIBUTIONS}

EC and SJ conceived and prepared the manuscript. MC, TT, AM, and KA contributed to essential input and manuscript editing.

\section{ACKNOWLEDGMENTS}

EC and SJ were supported by the Research University Grant for Individual (RUI, Grant No: 1001/CIPPT/8012230). MC was funded by the Research University Grant for Individual (RUI, Grant No: 1001/CIPPT/ 80110095). TT was supported by Fundamental Research Grant Scheme (FRGS, Grant No: 203/CIPPT/6711628) and TransDisciplinary Research Grant Scheme (TRGS, Grant No: 203/CIPPT/6769003). KA was supported by Universiti Sains Malaysia Post-Doctoral Fellowship. 


\section{REFERENCES}

1. Aras S, Zaidi MR. TAMeless traitors: macrophages in cancer progression and metastasis. Br J Cancer. (2017) 117:1583-91. doi: 10.1038/bjc.2017.356

2. Wynn TA, Chawla A, Pollard JW. Macrophage biology in development, homeostasis and disease. Nature. (2013) 496:445-55. doi: $10.1038 /$ nature 12034

3. van Dalen FJ, van Stevendaal MHME, Fennemann FL, Verdoes M, Ilina O. Molecular repolarisation of tumour-associated macrophages. Molecules. (2018) 24:E9. doi: 10.3390/molecules24010009

4. Wang LX, Zhang SX, Wu HJ, Rong XL, Guo J. M2b macrophage polarization and its roles in diseases. J Leukoc Biol. (2018) 106:345-58. doi: 10.1002/JLB.3RU1018-378RR

5. Biswas SK, Mantovani A. Macrophage plasticity and interaction with lymphocyte subsets: cancer as a paradigm. Nat Immunol. (2010) 11:889-96. doi: 10.1038/ni.1937

6. Zheng X, Turkowski K, Mora J, Brüne B, Seeger W, Weigert A, et al. Redirecting tumor-associated macrophages to become tumoricidal effectors as a novel strategy for cancer therapy. Oncotarget. (2017) 8:48436-52. doi: 10.18632/oncotarget.17061

7. Mills C. M1 and M2 macrophages: oracles of health and disease. Crit Rev Immunol. (2012) 32:463-88. doi: 10.1615/CritRevImmunol.v32.i6.10

8. Honkanen TJ, Tikkanen A, Karihtala P, Mäkinen M, Väyrynen JP, Koivunen JP. Prognostic and predictive role of tumour-associated macrophages in HER2 positive breast cancer. Sci Rep. (2019) 9:1-9. doi: 10.1038/s41598-019-47375-2

9. Lin $\mathrm{Y}, \mathrm{Xu}$ J, Lan H. Tumor-associated macrophages in tumor metastasis: biological roles and clinical therapeutic applications. J Hematol Oncol. (2019) 12:76. doi: 10.1186/s13045-019-0760-3

10. Hallam SL, Candido J, Coutinho R, Maniati E, Thompson RG, van Rooijen $\mathrm{N}$, et al. Manipulating tumor associated macrophages (TAM) in a mouse model of B-cell non-hodgkin lymphoma (NHL). Blood. (2011) 118:1657. doi: 10.1182/blood.V118.21.1657.1657

11. Cassetta L, Noy R, Swierczak A, Sugano G, Smith H, Wiechmann L, et al. Isolation of mouse and human tumor-associated macrophages. Adv Exp Med Biol. (2016) 899:211-29. doi: 10.1007/978-3-319-26666-4_12

12. Qiu SQ, Waaijer S, Zwager MC, de Vries EG, van der Vegt B, Schröder CP. Tumor-associated macrophages in breast cancer: innocent bystander or important player? Cancer Treat Rev. (2018) 70:178-89. doi: 10.1016/j.ctrv.2018.08.010

13. Li J, Xie Y, Wang X, Li F, Li S, Li M, et al. Prognostic impact of tumorassociated macrophage infiltration in esophageal cancer: a meta-analysis. Future Oncol. (2019) 15:2303-17. doi: 10.2217/fon-2018-0669

14. Yuan X, Zhang J, Li D, Mao Y, Mo F, Du W, et al. Prognostic significance of tumor-associated macrophages in ovarian cancer: a meta-analysis. Gynecol Oncol. (2017) 147:181-7. doi: 10.1016/j.ygyno.2017.07.007

15. Di Caro G, Cortese N, Castino GF, Grizzi F, Gavazzi F, Ridolfi C, et al. Dual prognostic significance of tumour-associated macrophages in human pancreatic adenocarcinoma treated or untreated with chemotherapy. Gut. (2016) 65:1710-20. doi: 10.1136/gutjnl-2015-309193

16. Yu M, Guan R, Hong W, Zhou Y, Lin Y, Jin H, et al. Prognostic value of tumorassociated macrophages in pancreatic cancer: a meta-analysis. Cancer Manag Res. (2019) 11:4041-58. doi: 10.2147/CMAR.S196951

17. Zhao X, Qu J, Sun Y, Wang J, Liu X, Wang F, et al. Prognostic significance of tumor-associated macrophages in breast cancer: a meta-analysis of the literature. Oncotarget. (2017) 8:30576-86. doi: 10.18632/oncotarget.15736

18. Wu P, Wu D, Zhao L, Huang L, Chen G, Shen G, et al. Inverse role of distinct subsets and distribution of macrophage in lung cancer prognosis: a meta-analysis. Oncotarget. (2016) 7:40451-60. doi: 10.18632/oncotarget.9625

19. Wang $\mathrm{X}$, Jiang J, Wu C. Prognostic significance of tumor-associated macrophage infiltration in gastric cancer: a meta-analysis. Genet Mol Res. (2016) 15:gmr15049040. doi: 10.4238/gmr15049040

20. Yagnik G, Rutowski MJ, Shah SS, Aghi MK. Stratifying nonfunctional pituitary adenomas into two groups distinguished by macrophage subtypes. Oncotarget. (2019) 10:2212-23. doi: 10.18632/oncotarget.26775

21. Dong P, Ma L, Liu L, Zhao G, Zhang S, Dong L, et al. CD86 ${ }^{+} / \mathrm{CD} 206^{+}$, diametrically polarized tumor-associated macrophages, predict hepatocellular carcinoma patient prognosis. Int J Mol Sci. (2016) 17:320. doi: 10.3390/ijms17030320
22. Klingen TA, Chen Y, Aas H, Wik E, Akslen LA. Tumor-associated macrophages are strongly related to vascular invasion, non-luminal subtypes, and interval breast cancer. Hum Pathol. (2017) 69:72-80. doi: 10.1016/j.humpath.2017.09.001

23. Jeong H, Hwang I, Kang SH, Shin HC, Kwon SY. Tumor-associated macrophages as potential prognostic biomarkers of invasive breast cancer. $J$ Breast Cancer. (2019) 22:38-51. doi: 10.4048/jbc.2019.22.e5

24. Mantovani A, Allavena P. The interaction of anticancer therapies with tumor-associated macrophages. J Exp Med. (2015) 212:435-45. doi: 10.1084/jem.20150295

25. Zhang W, Zhu XD, Sun HC, Xiong YQ, Zhuang PY, Xu HX, et al. Depletion of tumor-associated macrophages enhances the effect of sorafenib in metastatic liver cancer models by antimetastatic and antiangiogenic effects. Clin Cancer Res. (2010) 16:3420-30. doi: 10.1158/1078-0432.CCR-09-2904

26. Hughes R, Qian BZ, Rowan C, Muthana M, Keklikoglou I, Olson OC, et al. Perivascular M2 Macrophages Stimulate Tumor Relapse after Chemotherapy. Cancer Res. (2015) 75:3479-91. doi: 10.1158/0008-5472.CAN-14-3587

27. Yang C, He L, He P, Liu Y, Wang W, He Y, et al. Increased drug resistance in breast cancer by tumor-associated macrophages through IL-10/STAT3/bcl-2 signaling pathway. Med Oncol. (2015) 32:352. doi: 10.1007/s12032-014-0352-6

28. Xu M, Liu M, Du X, Li S, Li H, Li X, et al. Intratumoral delivery of IL21 overcomes anti-Her2/Neu resistance through shifting tumor-associated macrophages from M2 to M1 phenotype. J Immunol. (2015) 194:4997-5006. doi: 10.4049/jimmunol.1402603

29. Yin S, Huang J, Li Z, Zhang J, Luo J, Lu C, et al. The prognostic and clinicopathological significance of tumor-associated macrophages in patients with gastric cancer: a meta-analysis. PLoS ONE. (2017) 12:e0170042. doi: 10.1371/journal.pone.0170042

30. Zhong X, Chen B, Yang Z. The role of tumor-associated macrophages in colorectal carcinoma progression. Cell Physiol Biochem. (2018) 45:356-65. doi: $10.1159 / 000486816$

31. Wu MH, Lee WJ, Hua KT, Kuo ML, Lin MT. Macrophage infiltration induces gastric cancer invasiveness by activating the $\beta$-catenin pathway. PLOS ONE. (2015) 10:e0134122. doi: 10.1371/journal.pone.0134122

32. Zhang J, Yan Y, Yang Y, Wang L, Li M, Wang J, et al. High infiltration of tumorassociated macrophages influences poor prognosis in human gastric cancer patients, associates with the phenomenon of EMT. Medicine. (2016) 95:e2636. doi: 10.1097/MD.0000000000002636

33. Osinsky S, Bubnovskaya L, Ganusevich I, Kovelskaya A, Gumenyuk L, Olijnichenko G, et al. Hypoxia, tumour-associated macrophages, microvessel density, VEGF and matrix metalloproteinases in human gastric cancer: interaction and impact on survival. Clin Transl Oncol. (2011) 13:133-8. doi: 10.1007/s12094-011-0630-0

34. Wang B, Xu D, Yu X, Ding T, Rao H, Zhan Y, et al. Association of intratumoral infiltrating macrophages and regulatory $\mathrm{T}$ cells is an independent prognostic factor in gastric cancer after radical resection. Ann Surg Oncol. (2011) 18:2585-93. doi: 10.1245/s10434-011-1609-3

35. Lin CN, Wang CJ, Chao YJ, Lai MD, Shan YS. The significance of the coexistence of osteopontin and tumor-associated macrophages in gastric cancer progression. BMC Cancer. (2015) 15:128. doi: 10.1186/s12885-015-1114-3

36. Cui YL, Li HK, Zhou HY, Zhang T, Li Q. Correlations of tumor-associated macrophage subtypes with liver metastases of colorectal cancer. Asian Pac J Cancer Prev. (2013) 14:1003-7. doi: 10.7314/APJCP.2013.14.2.1003

37. Pantano F, Berti P, Guida FM, Perrone G, Vincenzi B, Amato MMC, et al. The role of macrophages polarization in predicting prognosis of radically resected gastric cancer patients. J Cell Mol Med. (2013) 17:1415-21. doi: $10.1111 /$ jcmm.12109

38. Zhang QW, Liu L, Gong CY, Shi HS, Zeng YH., Wang, XZ, et al. Prognostic significance of tumor-associated macrophages in solid tumor: a meta-analysis of the literature. PLoS ONE. (2012) 7:e50946. doi: 10.1371/journal.pone.0050946

39. Petrillo M, Zannoni GF, Martinelli E, Anchora LP, Ferrandina G, Tropeano $\mathrm{G}$, et al. Polarisation of tumor-associated macrophages toward M2 phenotype correlates with poor response to chemoradiation and reduced survival in patients with locally advanced cervical cancer. PLoS ONE. (2015) 10:e0136654. doi: 10.1371/journal.pone.0136654

40. Zhang M, He Y, Sun X, Li Q, Wang W, Zhao A, et al. A high M1/M2 ratio of tumor-associated macrophages is associated with extended survival 
in ovarian cancer patients. J Ovarian Res. (2014) 7:19. doi: 10.1186/17572215-7-19

41. Barros MH, Segges P, Vera-Lozada G, Hassan R, Niedobitek G. Macrophage polarization reflects $\mathrm{T}$ cell composition of tumor microenvironment in pediatric classical Hodgkin lymphoma and has impact on survival. PLoS ONE. (2015) 10:e0124531. doi: 10.1371/journal.pone.0124531

42. Chen X, Chen J, Zhang W, Sun R, Liu T, Zheng Y, et al. Prognostic value of diametrically polarized tumor-associated macrophages in multiple myeloma. Oncotarget. (2017) 8:112685-96. doi: 10.18632/oncotarget.22340

43. Barros MHM, Hauck F, Dreyer JH, Kempkes B, Niedobitek G. Macrophage polarisation: an immunohistochemical approach for identifying M1 and M2 macrophages. PLoS ONE. (2013) 8:e80908. doi: 10.1371/journal.pone.0080908

44. Dunstan RW, Wharton KA Jr, Quigley C, Lowe A. The use of immunohistochemistry for biomarker assessment-can it compete with other technologies? Toxicol Pathol. (2011) 39:988-1002. doi: $10.1177 / 0192623311419163$

45. Bertani FR, Mozetic P, Fioramonti M, Iuliani M, Ribelli G, Pantano F, et al. Classification of M1/M2-polarized human macrophages by label-free hyperspectral reflectance confocal microscopy and multivariate analysis. Sci Rep. (2017) 7:8965. doi: 10.1038/s41598-017-08121-8

46. Specht H, Emmott E, Perlman DH, Koller A, Slavov N. High-throughput single-cell proteomics quantifies the emergence of macrophage heterogeneity. bioRxiv. (2019) 665307. doi: 10.1101/665307

47. Kim SW, Roh J, Park CS. Immunohistochemistry for pathologists: protocols, pitfalls, and tips. J Pathol Transl Med. (2016) 50:411-8. doi: $10.4132 / j p t m .2016 .08 .08$

48. Stack EC, Wang C, Roman KA, Hoyt CC. Multiplexed immunohistochemistry, imaging, and quantitation: a review, with an assessment of Tyramide signal amplification, multispectral imaging and multiplex analysis. Methods. (2014) 70:46-58. doi: 10.1016/j.ymeth.2014.08.016

49. Kumar AT, Knops A, Swendseid B, Martinez-Outschoorn UE, Harshyne L, Philp NJ, et al. Prognostic significance of tumor-associated macrophage content in head and neck squamous cell carcinoma: a meta-analysis. Front Oncol. (2019) 9:656. doi: 10.3389/fonc.2019.00656

50. Pinto ML, Rios E, Durães C, Ribeiro R, Machado JC, Mantovani A, et al. The two faces of tumor-associated macrophages and their clinical significance in colorectal cancer. Front Immunol. (2019) 10:1875. doi: 10.3389/fimmu.2019.01875

51. Sica A, Mantovani A. Macrophage plasticity and polarization: in vivo veritas. J Clin Invest. (2012) 122:787-95. doi: 10.1172/JCI59643

52. Viola A, Munari F, Sanchez-Rodriguez R, Scolaro T, Castegna A. The metabolic signature of macrophage responses. Front Immunol. (2019) 10:1462. doi: 10.3389/fimmu.2019.01462

53. Helm O, Held-Feindt J, Schäfer H, Sebens S. M1 and M2: there is no "good" and "bad"-How macrophages promote malignancyassociated features in tumorigenesis. Oncoimmunology. (2014) 3:e946818. doi: 10.4161/21624011.2014.946818

54. Nathan C. Role of iNOS in human host defense. Science. (2006) 312:1874-5. doi: $10.1126 /$ science. $312.5782 .1874 \mathrm{~b}$

55. Ley K. M1 means kill; M2 means heal. J Immunol. (2017) 199:2191-3. doi: 10.4049/jimmunol.1701135

56. Rauch I, Müller M, Decker T. The regulation of inflammation by interferons and their STATs. JAKSTAT. (2013) 2:e23820. doi: 10.4161/jkst. 23820

57. Roszer T. Understanding the mysterious M2 macrophage through activation markers and effector mechanisms. Mediators Inflamm. (2015) 2015:816460. doi: $10.1155 / 2015 / 816460$

58. Stifano G, Christmann RB. Macrophage involvement in systemic sclerosis: do we need more evidence? Curr Rheumatol Rep. (2016) 18:2. doi: 10.1007/s11926-015-0554-8

59. Jiang T, Cheng X, Jia Y. Prognostic value of tumor-associated macrophages in classic Hodgkin's lymphoma: systematic review and meta-analysis. Int J Clin Exp Med. (2016) 9:10784-92.

60. Hu JM, Liu K, Liu JH, Jiang XL, Wang XL, Chen YZ, et al. CD163 as a marker of M2 macrophage, contribute to predict aggressiveness and prognosis of Kazakh esophageal squamous cell carcinoma. Oncotarget. (2017) 8:21526-38. doi: 10.18632/oncotarget.15630
61. Wang H, Hu WM, Xia ZJ, Liang Y, Lu Y, Lin SX, et al. High numbers of CD163+ tumor-associated macrophages correlate with poor prognosis in multiple myeloma patients receiving bortezomib-based regimens. J Cancer. (2019) 10:3239-45. doi: 10.7150/jca.30102

62. Wynn TA, Barron L. Macrophages: master regulators of inflammation and fibrosis. Semin Liver Dis. (2010) 30:245-57. doi: 10.1055/s-0030-1255354

63. Almatroodi SA, McDonald CF, Darby IA, Pouniotis DS. Characterization of M1/M2 tumour-associated macrophages (TAMs) and Th1/Th2 cytokine profiles in patients with NSCLC. Cancer Microenviron. (2016) 9:1-11. doi: 10.1007/s12307-015-0174-x

64. Dumars C, Ngyuen JM, Gaultier A, Lanel R, Corradini N, Gouin F, et al. Dysregulation of macrophage polarization is associated with the metastatic process in osteosarcoma. Oncotarget. (2016) 7:78343-54. doi: 10.18632/oncotarget.13055

65. Marchesi F, Cirillo M, Bianchi A, Gately M, Olimpieri OM, Cerchiara E, et al. High density of CD68+/CD163+ tumour-associated macrophages (M2TAM) at diagnosis is significantly correlated to unfavorable prognostic factors and to poor clinical outcomes in patients with diffuse large B-cell lymphoma. Hematol Oncol. (2015) 33:110-2. doi: 10.1002/hon.2142

66. Rakaee M, Busund LTR, Jamaly S, Paulsen EE, Richardsen E, Andersen S, et al. Prognostic value of macrophage phenotypes in resectable non-small cell lung cancer assessed by multiplex immunohistochemistry. Neoplasia. (2019) 21:282-93. doi: 10.1016/j.neo.2019.01.005

67. Drew CP, Shieh WJ. Immunohistochemistry. In: Rupprecht C, Nagarajan T, editors. Current Laboratory Techniques in Rabies Diagnosis, Research and Prevention. Vol. 2. Cambridge, MA: Academic Press (2015). doi: 10.1016/B978-0-12-801919-1.00010-5

68. Sousa S, Brion R, Lintunen M, Kronqvist P, Sandholm J, Mönkkönen J, et al. Human breast cancer cells educate macrophages toward the M2 activation status. Breast Cancer Res. (2015) 17:101. doi: 10.1186/s13058-015-0621-0

69. Shu QH, Ge YS, Ma HX, Gao XQ, Pan JJ, Liu D, et al. Prognostic value of polarized macrophages in patients with hepatocellular carcinoma after curative resection. J Cell Mol Med. (2016) 20:1024-35. doi: $10.1111 / \mathrm{jcmm} .12787$

70. Lúcio PS, Ribeiro DC, Aguiar MC, Alves PM, Nonaka CF, Godoy GP. Tumor-associated macrophages (TAMs): clinical-pathological parameters in squamous cell carcinomas of the lower lip. Braz. Oral Res. (2016) 30:e95. doi: 10.1590/1807-3107BOR-2016.vol30.0095

71. Lin MW, Yang CY, Kuo SW, Wu CT, Chang YL, Yang PC. The prognostic significance of pSTAT1 and CD163 expressions in surgically resected stage 1 pulmonary squamous cell carcinomas. Ann Surg Oncol. (2016) 23:3071-81. doi: 10.1245/s10434-016-5244-x

72. Xu L, Zhu Y, Chen L, An H, Zhang W, Wang G, et al. Prognostic value of diametrically polarized tumor-associated macrophages in renal cell carcinoma. Ann Surg Oncol. (2014) 21:3142-50. doi: 10.1245/s10434-014-3601-1

73. Kelly MG, Francisco AM, Cimic A, Wofford A, Fitzgerald NC, Yu J, et al. Type 2 endometrial cancer is associated with a high density of tumor-associated macrophages in the stromal compartment. Reprod Sci. (2015) 22:948-53. doi: $10.1177 / 1933719115570912$

74. Yu F, Gong L, Mo Z, Wang W, Wu M, Yang J, et al. Programmed death ligand1 , tumor infiltrating lymphocytes and HLA expression in Chinese extrahepatic cholangiocarcinoma patients: possible immunotherapy implications. Biosci Trends. (2019) 13:58-69. doi: 10.5582/bst.2019.01003

75. Lee C, Lee J, Choi SA, Kim SK, Wang KC, Park SH, et al. M1 macrophage recruitment correlates with worse outcome in SHH Medulloblastomas. BMC Cancer. (2018) 18:535. doi: 10.1186/s12885-018-4457-8

76. Mori K, Haraguchi S, Hiori M, Shimada J, Ohmori Y. Tumor-associated macrophages in oral premalignant lesions coexpress CD163 and STAT1 in a Th1-dominated microenvironment. BMC Cancer. (2015) 15:573. doi: 10.1186/s12885-015-1587-0

77. Buitrago DH, Patnaik SK, Kadota K, Kannisto E, Jones DR, Adusumilli PS. Small RNA sequencing for profiling microRNAs in long-term preserved formalin-fixed and paraffin-embedded non-small cell lung cancer tumor specimens. PLoS ONE. (2015) 10:e0121521. doi: 10.1371/journal.pone.0121521

78. Smith C. FFPE or Frozen? Working with Human Clinical Samples (2014). Available online at: https://www.biocompare.com/Editorial-Articles/168948- 
FFPE- or-Frozen-Working-with-Human-Clinical-Samples/

(accessed August 18, 2019).

79. Yang M, Li Z, Ren M, Li S, Zhang L, Zhang X, et al. Stromal infiltration of tumor-associated macrophages conferring poor prognosis of patients with basal-like breast carcinoma. J Cancer. (2018) 9:2308-16. doi: $10.7150 /$ jca. 25155

80. Shi SR, Liu C, Taylor CR. Standardization of immunohistochemistry for formalin-fixed, paraffin-embedded tissue sections based on the antigenretrieval technique: from experiments to hypothesis. J Histochem Cytochem. (2007) 55:105-9. doi: 10.1369/jhc.6P7080.2006

81. Leong TY, Cooper K, Leong AS. Immunohistology-past, present, and future. Adv Anat Pathol. (2010) 17:404-18. doi: 10.1097/PAP.0b013e3181 f8957c

82. Lau SK, Chu PG, Weiss LM. CD163A specific marker of macrophages in paraffin-embedded tissue samples. Am J Clin Pathol. (2004) 122:794-801. doi: 10.1309/QHD6YFN81KQXUUH6

83. Mestas J, Hughes CC. Of mice and not men: differences between mouse and human immunology. J Immunol. (2004) 172:2731-8. doi: 10.4049/jimmunol.172.5.2731

84. Schneemann M, Schoeden G. Macrophage biology and immunology: man is not a mouse. J Leukoc Biol. (2007) 81:579. doi: 10.1189/jlb. 1106702

85. Gerrick KY, Gerrick ER, Gupta A, Wheelan SJ, Yegnasubramanian S, Jaffee EM. Transcriptional profiling identifies novel regulators of macrophage polarization. PLoS ONE. (2018) 13:e0208602. doi: 10.1371/journal.pone.0208602

86. Hamilton PW, Van Diest PJ, Williams R, Gallagher AG. Do we see what we think we see? The complexities of morphological assessment. J Pathol. (2009) 218:285-91. doi: 10.1002/path.2527

87. Tuominen VJ, Ruotoistenmäki S, Viitanen A, Jumppanen M, Isola J. ImmunoRatio: a publicly available web application for quantitative image analysis of estrogen receptor (ER), progesterone receptor (PR), and Ki-67. Breast Cancer Res. (2010) 12:R56. doi: 10.1186/bcr2615

Conflict of Interest: The authors declare that the research was conducted in the absence of any commercial or financial relationships that could be construed as a potential conflict of interest.

The handling Editor declared a shared affiliation, though no other collaboration, with the authors at time of review.

Copyright (C) 2020 Jayasingam, Citartan, Thang, Mat Zin, Ang and Ch'ng. This is an open-access article distributed under the terms of the Creative Commons Attribution License (CC BY). The use, distribution or reproduction in other forums is permitted, provided the original author(s) and the copyright owner(s) are credited and that the original publication in this journal is cited, in accordance with accepted academic practice. No use, distribution or reproduction is permitted which does not comply with these terms. 